IdeAs

Idées d'Amériques

15 | 2020

Eau et gestion de l'eau dans les Amériques

\title{
De la transition à l'explosion du 18-0 : malaise et rébellion populaire aux portes de la terre promise
}

Emmanuelle Barozet

\section{(2) OpenEdition}

1 Journals

Édition électronique

URL : http://journals.openedition.org/ideas/8354

DOI : $10.4000 /$ ideas.8354

ISSN : 1950-5701

Éditeur

Institut des Amériques

Référence électronique

Emmanuelle Barozet, « De la transition à l'explosion du 18-0 : malaise et rébellion populaire aux portes de la terre promise », IdeAs [En ligne], 15 | 2020, mis en ligne le 01 mars 2020, consulté le 10 décembre 2020. URL : http://journals.openedition.org/ideas/8354; DOI : https://doi.org/10.4000/ideas.8354

Ce document a été généré automatiquement le 10 décembre 2020.

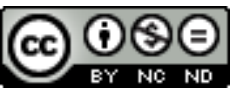

IdeAs - Idées d'Amériques est mis à disposition selon les termes de la licence Creative Commons Attribution - Pas d'Utilisation Commerciale - Pas de Modification 4.0 International. 


\title{
De la transition à l'explosion du 18- 0 : malaise et rébellion populaire aux portes de la terre promise
}

\author{
Emmanuelle Barozet
}

Je remercie le COES, CONICYT/FONDAP/15130009, les projet Fondecyt n 1160984 et 1190436, ainsi que le réseau INCASI du programme European Union's Horizon 2020 research and innovation programme under the Marie Skłodowska-Curie GA No 691004 coordonné par le Dr. Pedro LópezRoldán.

1 Nous réaliserons ici une analyse de moyen terme, dans le but de comprendre la violente crise qui secoue le Chili. Cette dernière montre un nouveau visage de ce territoire longiligne du Cône Sud: celui d'un petit pays de 17 millions d'habitants, très inégalitaire et qui, cependant, se percevait comme l'une des rares nations immunes à la violence structurelle du continent latino-américain. Partant des notions de cohésion ${ }^{1}$ et de contrat social ${ }^{2}$, nous reviendrons sur la mise en place de mécanismes de blocage institutionnel lors de la transition de la fin des années 1980. Ceux-ci ont empêché l'élargissement du processus décisionnel et limité la redistribution des fruits de la croissance. Malgré les succès macroéconomiques du Chili lors des trente dernières années, la distance grandissante entre les services des secteurs public et privé a renforcé la ségrégation sociale. Notre analyse vient compléter, dans ce même dossier, celle sur l'évolution de long terme de l'économie chilienne (voir article de Gárate) et anticipe celle consacrée à la conjoncture actuelle (voir article de Maillet et Faure), en nous concentrant sur la double dimension politique et sociale du processus historique récent.

\section{Les enclaves autoritaires après la transition : une société dépolitisée?}

2 Le Chili a été présenté durant les années 1990 comme un exemple de transition démocratique. À la suite des dix-sept ans de régime militaire, Augusto Pinochet quitte 
le pouvoir à reculons, mais sans laisser un pays instable du point de vue institutionnel. Imposée par un référendum biaisé, la Constitution de 1980 favorise le maintien de l'ordre et installe un cadre rigide et peu légitime. Mais à l'époque, les conditions ne sont pas réunies pour un nouveau contrat social, dans un pays où aucun des processus constitutionnels antérieurs n'a inclus la population. Cependant, la société semble s'accommoder de cette transition incomplète et d'une gestion des affaires publiques «dans la mesure du possible». Il convient de préciser qu'une partie de la classe politique et de l'opinion publique est alors durablement traumatisée non seulement par les années de dictature, mais aussi par les débordements populaires des années Allende (1970-1973).

3 Cependant, il existe de nombreuses critiques à cette transition élitiste (Garretón, M. 2006), que ce soit du point de vue des défenseurs des droits humains, des secteurs populaires, des peuples indigènes ou des représentants des droits des femmes et des minorités : le système sera verrouillé jusqu'en 1998, quand Augusto Pinochet est arrêté à Londres. Commence alors une prise de conscience des nombreuses anomalies de la transition et la fin d'une première étape en 2005 , avec d'importants amendements à la constitution de 1980. La découverte après 2001 de la corruption de la famille Pinochet finit par sceller la sortie des militaires de la vie politique, mais la constitution de 1980, malgré ses amendements, reste illégitime pour une partie de la population.

En ce qui concerne la cohésion sociale, la question se concentre sur la moitié de la population en situation de pauvreté au moment de la transition. Les gouvernements de la Démocratie Chrétienne qui se succèdent au pouvoir (Patricio Aylwin de 1990 à 1994 et Eduardo Frei de 1994 à 2000) réalisent d'importants investissements dans l'éducation, la santé et l'emploi. Le système d'impôts n'étant ni solidaire, ni redistributif, les dépenses publiques sont alimentées par le super cycle de cuivre, qui voit son prix multiplié par deux par rapport à la tendance historique $\mathrm{du} \mathrm{xx}^{\mathrm{e}}$ siècle, ce qui permet d'assurer une manne importante pour l'État entre 1990 et 2014. La pauvreté baisse effectivement de manière importante (de $45 \%$ à moins de $20 \%$ lors de cette décennie), ce qui permet de maintenir un certain degré de calme social, sinon de cohésion, alors que le revenu per capita augmente à 13.000 dollars en 2010. La possibilité d'accéder bientôt au club des pays riches ou mieux encore, des pays développés, se retrouve dans la rhétorique de tous les gouvernements, alors que s'installe un discours centré sur la méritocratie. La terre promise de la prospérité est en vue. En parallèle, la baisse progressive de la participation politique et en particulier de celle des jeunes semble ancrer l'idée que l'on entre dans une " démocratie de faible intensité ».

\section{Les mobilisations des années 2000 : la question des inégalités}

5 Cependant ces chiffres positifs masquent de profondes inégalités. Ces succès économiques sont incontestables, surtout lorsque l'on compare le Chili avec ses voisins. Ce petit pays ouvert aux exportations connaitra trente ans de croissance économique, un record en Amérique latine, où les cycles expansifs ne durent en général pas plus de dix ans. Mais en toile de fond, les revendications historiques des peuples indigènes sont étouffées par une forte politique de répression, puis de militarisation, du fait que leurs demandes s'opposent à l'exploitation des ressources naturelles. Ce conflit sera toutefois amplifié par les mouvements de protection de l'environnement qui se développent 
dans les années 2000. En effet, le système économique "extractiviste " accroît l'exploitation des ressources naturelles, alors que l'expansion urbaine sans planification ne permet pas l'amélioration significative des conditions de vie d'amples secteurs populaires. Sous le gouvernement de Eduardo Frei (1994-2000), puis du socialiste Ricardo Lagos (2000-2006), la modernisation du système politique s'intensifie. Dans le même temps, la baisse de la pauvreté se stabilise autour de $20 \%$. Un nouveau récit social remplace alors celui de la réduction de la pauvreté : l'attention se porte sur les inégalités, phénomène visible au niveau international, mais qui s'exprime au Chili de manière très forte, du fait d'un système de protection sociale peu développé et d'une forte accumulation des richesses en haut de la société (Espinoza V. et E. Barozet, 2019a). La cohésion sociale jusqu'alors structurée autour de relations verticales plus qu'horizontales entre les membres de la société et l'État, montre clairement des trouées béantes dans la gestion du bien commun, alors que le manque de confiance interpersonnelle et interinstitutionnelle, commune en Amérique latine, érode le modèle social .

6 En 2001, les lycéens organisent les premières manifestations nationales, avec le soutien des syndicats d'enseignants. Cet épisode (le Mochilazo), ouvre une nouvelle étape de politisation de la population. En 2006, se structure un ample mouvement pour le droit à l'éducation, qui fédère les lycéens contre la privatisation du système scolaire. La révolution des Pingouins (en référence à l'uniforme scolaire) est l'une des mobilisations les plus intenses depuis le retour à la démocratie. Les négociations entamées par le gouvernement socialiste de Michelle Bachelet (2006-2010) désamorcent en partie le conflit grâce à la mise en place d'une commission technique, stratégie que les gouvernements postérieurs utiliseront aussi en cas de conflit social. Finalement, les lycéens n'obtiendront pas une réforme du système scolaire, mais la question de la qualité de l'enseignement et des inégalités est posée de manière durable, ainsi que celle de la fracture sociale croissante entre ceux qui accèdent à des services de qualité dans le privé, et les autres, la majorité.

7 D'autres mouvements sociaux pointent de nouvelles limites du modèle de développement. Sur la question territoriale par exemple, les revendications portent désormais sur une demande de plus forte autogestion et décentralisation. Les associations de défense des quartiers face aux investissements urbains de grande envergure ou encore les ONG de protection de l'environnement contre les grandes entreprises minières et du secteur de l'énergie, inaugurent un nouveau répertoire d'action. Il s'agit de mettre en jeu des compétences techniques et expertes dans une trame réglementaire et judicaire plus dense (Biskupovic, C., 2017). Le malaise, que certains avaient analysé jusqu'alors en termes culturels liés aux paradoxes de la modernisation économique (PNUD, 1998), se transforme en revendications pour un accès égalitaire à des services de qualité et de reconnaissance de droits. S'ouvre ainsi un nouvel horizon social et politique face à un présent qui ne remplit plus les aspirations de nouvelles générations sorties de la pauvreté et que l'on labellise comme des classes moyennes, sans leur donner toutefois la stabilité et les revenus qu'impliquent l'imaginaire de la moyennisation. 


\section{Les entraves du système politique : des réponses incomplètes et tardives}

8 Au-delà du problème des enclaves de la transition et du déficit de participation, s'ajoute un manque important de représentation des demandes sociales. En effet, le taux de syndicalisation ne dépasse pas les $20 \%$, tout en étant concentré dans les secteurs les plus productifs comme les mines ou la banque. Les conflits dans le monde du travail s'émancipent de plus en plus du cadre juridique, au point que les grèves illégales deviennent plus nombreuses que les grèves légales (Observatorio de Huelgas Laborales, 2019).

9 La déliquescence des institutions publiques s'accentue durant les années 2010 (sur la réforme administrative, voir article de Egaña et Pliscoff dans ce dossier). L'adoption du vote volontaire en 2012 avait déjà érodé la participation électorale, qui passe sous la barre des $40 \%$ lors des élections municipales de 2016. Les scandales de pédophilie qui éclatent en 2010 au sein de l'Église sont un premier choc. Les affaires de corruption qui affectent ensuite en 2015 les partis de l'ensemble du spectre politique rendent brutalement obsolète l'idée que le Chili, du fait de sa culture légaliste, serait immune à l'affaiblissement de la morale publique. Finalement, la même année, un nouveau scandale de grande ampleur est mis au jour au sein des forces armées et des Carabiniers, qui ont soustrait, durant des années, des sommes très importantes, pour leur propre enrichissement. L'opinion publique devient alors très critique à l'égard de ses principales institutions, dans un contexte international de crise des formes classiques de la représentation politique (CEP, 2020).

Malgré l'arrivée au Parlement en 2013 puis en 2017 de jeunes issus du mouvement pour l'éducation, les pratiques traditionnelles comme le clientélisme redeviennent une manière importante de contrôle de la part des caudillos. Certains maires et parlementaires, du fait de l'absence d'interdiction du cumul des mandats, s'enkystent au pouvoir ; ce qui, dans certains cas, crée des zones de capture de l'État et de l'intérêt public (Espinoza V. et E. Barozet, 2019b). Finalement, une grande partie de la population se sent de moins en moins identifiée à l'État, les services publics comme l'éducation et la santé étant considérés comme de mauvaise qualité. Dès qu'elles le peuvent, les familles migrent vers le secteur privé, au prix d'un endettement toujours plus élevé. Sans pacte social renouvelé et face à un manque flagrant de cohésion sociale, la pression monte.

\section{L'intensification des mobilisations dans les années 2010}

L'absence de réforme profonde des aspects les plus discriminatoires du système économique, politique et social, malgré un calme apparent, finit par motiver un nouveau mouvement social de grande ampleur : celui des étudiants en 2011. En termes d'opportunités politiques, le premier gouvernement de droite qui arrive au pouvoir depuis la transition, celui de Sebastián Piñera (2010-2014), déclenche des critiques plus vives contre le modèle technocratique (voir article de Larrouqué dans ce dossier), le gouvernement des experts et de l'argent. 2010 ayant été marqué par un tremblement de terre, ça n'est que l'année suivante que les étudiants redescendent dans la rue. Il 
s'agit en partie de la génération de 2001 et 2006, qui a transité du secondaire à l'université et qui se trouve face à un endettement important. En effet, l'explosion du tertiaire, qui passe de 200000 à 1300000 personnes entre 1990 et 2018 se fait en grande partie par le financement des familles. Le soutien ambigu d'une grande partie de la population n'ouvre toutefois pas la voie à une réforme profonde de l'éducation. Cependant, l'élargissement de l'accès à l'enseignement supérieur est permis grâce à un système de bourses. Au demeurant, l'insertion postérieure sur le marché du travail n'en est pas pour autant garantie. Le problème est à nouveau repoussé ; mais cette fois-ci, les commissions techniques ne sont plus suffisantes.

Un nouveau mouvement contre le système des retraites se constitue en $2013(\mathrm{No}+\mathrm{AFP})$, créant un double front de manifestants : aux jeunes, s'ajoutent les personnes âgées qui bravent des conditions misérables de passage à la retraite. Le deuxième gouvernement de Michelle Bachelet (2014-2018) décide d'entamer un large programme de réformes ayant pour objectif de prendre en charge les inégalités les plus criantes. Cependant, le scandale qui affecte la famille de la présidente en 2015, ainsi que l'ampleur des réformes traitées par le parlement, aboutissent à ce que les mesures phares soient abandonnées en cours de route (réforme à la Constitution, réforme des impôts et du système de santé). L'absence de renouvellement du personnel politique permet à nouveau l'alternance, lors du retour au pouvoir de Sebastián Piñera (2018-2022), avec un programme fondé, en grande partie, sur la promotion de la croissance économique. Mais la lune de miel est de très courte durée : en 2018, le mouvement féministe secoue à nouveau les bases du système économique et politique chilien (voir article de Ponce dans ce dossier). L'essoufflement du modèle d'exportation, la baisse de la croissance en Chine (et donc la moindre demande de cuivre), sur fond de guerre commerciale sinoaméricaine, finissent par enrayer durablement la machine économique, laquelle se grippe en 2019. Le discrédit de la classe politique, la mauvaise gestion des attentes de l'opinion publique, ainsi que le mépris visible des élites gouvernementales, déclenchent finalement le processus de furie sociale qui s'abat dans tout le pays à partir du 18 octobre. Déjà résiduelle, la cohésion sociale s'en trouve pulvérisée, alors qu'émerge une demande structurelle forte: une nouvelle constitution pour refonder les bases de la société (voir article de Escudero et Gajardo dans ce dossier).

\section{Conclusion}

Les crises sociales comme celle que connaît le Chili sont probables dans une certaine mesure, mais ni leur ampleur ni leur timing ne sont prévisibles, d'autant que l'hypothèse de l'apathie politique et de l'acceptation des inégalités avaient donné l'image d'un pays démobilisé. Selon The Economist (2019), les manifestations qui agitent le Chili depuis octobre 2019 sont un signe de démocratisation. Mais les difficultés de la classe politique pour surmonter cette crise se répètent: résistance au changement d'une part importante de l'élite, qui a recours à la répression pour endiguer sans succès le mouvement social (voir article d'Aguilera dans ce dossier), fragmentation de la gauche qui ne sait pas comment gérer le processus de concertation nationale qui doit aboutir à une nouvelle constitution), et finalement un ras-le-bol d'une partie des citoyens tentés par le mantra populiste "que se vayan todos".

Contrat social et bases de la cohésion sont aujourd'hui l'objet d'un ample débat public. La rébellion populaire atteste d'une double crise : de la représentation d'une part et du 
modèle économique d'autre part. Malheureusement, dans un pays où les élites ne sont pas prêtes à reconnaître l'autonomie des citoyens dans l'expression de la souveraineté nationale, l'élaboration d'un pacte qui « défende et protège de toute la force commune la personne et les biens de chaque associé » (Rousseau, J.J., 1792) soulève encore de nombreux doutes. Une nouvelle terre promise est peut-être en vue.

\section{BIBLIOGRAPHIE}

Biskupovic, Consuelo, «Expertise et amour dans la défense de la nature », Revue SociologieS, 2017. Burchi, Francesco, Armin von Schiller et Christoph Strupart, Revenue Collection and Social Policies: Their Underestimated Contribution to Social Cohesion, Briefing paper, Bonn, Deutsche Institut für Entwicklungspolitik, 2020.

CEP, Estudio Nacional de Opinión Pública Diciembre 2019, Santiago, CEP, 2020.

Espinoza, Vicente et Emmanuelle Barozet, « Contention and Debates about Social Policies and Inequalities in Contemporary Chile » in Ewa Bogalska-Martin et Emmanuel Matteudi (eds.), The Social Question in the Global World. The Quest for an Effective Paradigm, Cambridge, Cambridge University Press, 2019a, p. 81-108.

Espinoza, Vicente et Emmanuelle Barozet, « Nepotismo, amiguismo y la rabia de los que no son de ningún lote », CIPER, 30 août 2019b, https://urlz.fr/bHYy, page consultée le 09/03/2020

Garretón, Manuel Antonio, « Reflexiones sobre la democratización política chilena », in Collectif, Brasil y Chile. Una mirada hacia América Latina, de Collectif, Santiago : RIL Ediciones, 2006, p. 45-72.

Latinobarómetro, Informe 2018, Santiago : Latinobarómetro, 2018.

Observatorio de Huelgas Laborales, Informe de huelgas laborales en Chile 2018. Santiago : UAH COES, 2019.

PNUD, Informe sobre Desarrollo Humano 1998. Las paradojas de la modernización. Santiago : PNUD, 1998.

Rousseau, Jean-Jacques, Du contrat social ou Principes du droit politique [1792], Paris, Flammarion, 2012.

The Economist, Democracy Index 2019, Londres, The Economist, https://urlz.fr/bIq9, page consultée le 09/03/2020

\section{NOTES}

1. «La cohésion sociale fait référence à la fois aux relations verticales et horizontales entre les membres de la société et l'État. Elle est caractérisée par un ensemble d'attitudes et de normes qui incluent la confiance, une identité inclusive et la coopération pour le bien commun » (Burchi, F., von Schiller, A. et C. Strupart, 2020). 
2. Le contrat ou pacte social est « une forme d'association qui défend et protège de toute la force commune la personne et les biens de chaque associé, et par laquelle chacun, s'unissant à tous, n'obéit pourtant qu'à lui-même, et reste aussi libre qu'auparavant » (Rousseau, J.J, 1792).

\section{AUTEUR}

\section{EMMANUELLE BAROZET}

Emmanuelle Barozet est affiliée au Département de Sociologie de l'Université du Chili / COES) ebarozet@uchile.cl 